International Journal of Pure and Applied Mathematics

Volume 92 No. 2 2014, 169-179

ISSN: 1311-8080 (printed version); ISSN: 1314-3395 (on-line version)

url: http://www.ijpam.eu

doi: http://dx.doi.org/10.12732/ijpam.v92i2.3

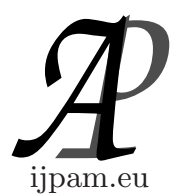

\title{
POSITIVE SOLUTIONS FOR BOUNDARY VALUE PROBLEM OF SINGULAR FRACTIONAL FUNCTIONAL DIFFERENTIAL EQUATION
}

\author{
Limei Song \\ School of Mathematics \\ Jiaying University \\ Meizhou, Guangdong, 514015, P.R. CHINA
}

\begin{abstract}
In this paper, we consider a class of singular fractional order functional differential equations with delay. By means of a fixed point theorem on cones, some sufficient conditions for the existence of at least one or two positive solutions for the boundary value problem are established.

We also give examples to illustrate the applicability of our main results.

AMS Subject Classification: 26A33, 34B18

Key Words: singular problem, fractional functional differential equation, boundary value problem, fixed point theorem, Positive solution
\end{abstract}

\section{Introduction}

Fractional differential equations have been of great interest recently. It is caused both by the intensive development of the theory of fractional calculus itself and by the applications (see[1], [2], [3], [4], [5]).

In [6], [7], [8], [9], [10], they studied the existence and multiplicity of positive solutions to fractional differential equations, and obtained some results. However, to the best of the author knowledge, there are few articles studying

Received: October 11, 2013

(c) 2014 Academic Publications, Ltd. url: www.acadpubl.eu 
the functional differential equations of fractional order. In [11], the authors studied the existence of positive solution for boundary value problem of fourthorder FDE. Motivated by the work above, this paper investigates the existence of positive solutions for singular fractional order functional differential equation with boundary conditions

$$
\left\{\begin{array}{l}
D_{0^{+}}^{\alpha} u(t)+r(t) f\left(u_{t}\right)=0, \quad 0<t<1, \quad 2<\alpha \leq 3 \\
u(t)=\phi(t), \quad-\tau \leq t \leq 0 \\
u(0)=u^{\prime}(0)=u^{\prime}(1)=0
\end{array}\right.
$$

where $D_{0^{+}}^{\alpha}$ is the standard Riemann-Liouville fractional derivative of order $\alpha ; \phi(t) \in C([-\tau, 0],[0,+\infty)), \phi(0)=0 ;$ for $t \in[0,1], u_{t}(\theta)=u(t+\theta), \theta \in$ $[-\tau, 0], 0 \leq \tau<\frac{1}{2}$ is a constant.

\section{Preliminaries}

Definition 1. ([6]) The Riemann-Liouville fractional derivative of order $\alpha>0$ of a continuous function $y:(0, \infty) \rightarrow \mathbf{R}$ is given by

$$
D_{0^{+}}^{\alpha} y(t)=\frac{1}{\Gamma(n-\alpha)}\left(\frac{\mathrm{d}}{\mathrm{d} t}\right)^{n} \int_{0}^{t} \frac{y(s)}{(t-s)^{\alpha-n+1}} \mathrm{~d} s,
$$

provided that the right side is pointwise defined on $(0, \infty)$, where $n=[\alpha]+1$, $[\alpha]$ denotes the integer part of the number $\alpha$.

Lemma 2. ([10]) Let $h(t) \in C[0,1]$ be a given function. Then the boundary value problem

$$
\left\{\begin{array}{l}
D_{0^{+}}^{\alpha} u(t)+h(t)=0, \quad 0<t<1,2<\alpha \leq 3, \\
u(0)=u^{\prime}(0)=u^{\prime}(1)=0,
\end{array}\right.
$$

has a unique solution

$$
u(t)=\int_{0}^{1} G(t, s) h(s) \mathrm{d} s,
$$

where

$$
G(t, s)=\left\{\begin{array}{lc}
\frac{t^{\alpha-1}(1-s)^{\alpha-2}-(t-s)^{\alpha-1}}{\Gamma(\alpha)}, & 0 \leq s \leq t \leq 1 \\
\frac{t^{\alpha-1}(1-s)^{\alpha-2}}{\Gamma(\alpha)}, & 0 \leq t \leq s \leq 1
\end{array}\right.
$$

Lemma 3. ([10]) The function $G(t, s)$ defined by (2) satisfies

(i) $G(t, s)>0, t, s \in(0,1)$; 
(ii) $G(t, s) \leq \max _{0 \leq t \leq 1} G(t, s)=G(1, s), t, s \in[0,1]$;

(iii) $\min _{\frac{1}{4} \leq t \leq \frac{3}{4}} G(t, s) \geq\left(\frac{1}{4}\right)^{\alpha-1} G(1, s), s \in(0,1)$.

Note that $\left(\frac{1}{4}\right)^{\alpha-1} \geq \frac{1}{16}$, so we have $\min _{\frac{1}{4} \leq t \leq \frac{3}{4}} G(t, s) \geq \frac{1}{16} G(1, s)$ for $s \in(0,1)$.

Let $C=C([-\tau, 0], \mathbf{R})$ be a Banach space with a norm $\|\varphi\|_{C}=\sup _{-\tau \leq \theta \leq 0}|\varphi(\theta)|$ and

$$
C^{+}=\{\varphi \in C ; \varphi(\theta) \geq 0, \theta \in[-\tau, 0]\} .
$$

Define $E=\left\{t \in[0,1]: \frac{1}{4} \leq t+\theta \leq \frac{3}{4},-\tau \leq \theta \leq 0\right\}=\left[\frac{1}{4}+\tau, \frac{3}{4}\right]$.

We assume the following:

$\left(A_{1}\right) \quad f$ is a nonnegative continuous functional defined on $C^{+}$, and $f$ maps bounded set in $C^{+}$into bounded set in $\mathbf{R}$.

$\left(A_{2}\right) \quad r(t)$ is a nonnegative measurable function defined on $[0,1]$, and satisfies

$$
0<\int_{E} G(1, s) r(s) \mathrm{d} s<\int_{0}^{1} G(1, s) r(s) \mathrm{d} s<+\infty .
$$

We would mention that $r(t)$ is allowed to be zero on some subset of $E$ and has singularity at points $t=0$ and $t=1$.

Suppose that $u(t)$ is a solution of $\operatorname{BVP}(1)$, then

$$
u(t)=\left\{\begin{array}{l}
\int_{0}^{1} G(t, s) r(s) f\left(u_{s}\right) \mathrm{d} s, \quad 0 \leq t \leq 1, \\
\phi(t), \quad-\tau \leq t \leq 0 .
\end{array}\right.
$$

Suppose that $\bar{x}(t)$ is the solution of $\operatorname{BVP}(1)$ with $f \equiv 0$, then

$$
\bar{x}(t)=\left\{\begin{array}{lr}
0, & 0 \leq t \leq 1, \\
\phi(t), & -\tau \leq t \leq 0 .
\end{array}\right.
$$

Let $x(t)=u(t)-\bar{x}(t)$, then we have from (3)and(4) that

$$
x(t)=\left\{\begin{array}{l}
\int_{0}^{1} G(t, s) r(s) f\left(x_{s}+\bar{x}_{s}\right) \mathrm{d} s, \quad 0 \leq t \leq 1, \\
0, \quad-\tau \leq t \leq 0 .
\end{array}\right.
$$

Let $K$ be a cone in Banach space $C[-\tau, 1]$ defined by

$$
K=\left\{x \in C[-\tau, 1] \mid x(t)=0, t \in[-\tau, 0] ; \min _{\frac{1}{4} \leq t \leq \frac{3}{4}} x(t) \geq \frac{1}{16}\|x\| ;\right.
$$




$$
x(t)>0, t \in(0,1)\}
$$

where $\|x\|:=\max \{|x(t)|:-\tau \leq t \leq 1\}$.

Define $T: K \rightarrow C[-\tau, 1]$ as

$$
(T x)(t)=\left\{\begin{array}{l}
\int_{0}^{1} G(t, s) r(s) f\left(x_{s}+\bar{x}_{s}\right) \mathrm{d} s, \quad 0 \leq t \leq 1 \\
0, \quad-\tau \leq t \leq 0
\end{array}\right.
$$

We define $\|x\|_{[0,1]}=\sup \{|x(t)| ; 0 \leq t \leq 1\}$, then we have $\|x\|=\|x\|_{[0,1]}$ and $\|T x\|=\|T x\|_{[0,1]}$ for any $x \in K$.

For $0<t<1, x \in K$, we obtain from (5) and Lemma 3 that $(T x)(t)>0$, and

$$
\begin{aligned}
&\|T x\| \leq \int_{0}^{1} \max _{0 \leq t \leq 1} G(t, s) r(s) f\left(x_{s}+\bar{x}_{s}\right) \mathrm{d} s \\
& \min _{\frac{1}{4} \leq t \leq \frac{3}{4}}(T x)(t)=\min _{\frac{1}{4} \leq t \leq \frac{3}{4}} \int_{0}^{1} G(t, s) r(s) f\left(x_{s}+\bar{x}_{s}\right) \mathrm{d} s \\
& \geq \frac{1}{16} \int_{0}^{1} \max _{0 \leq t \leq 1} G(t, s) r(s) f\left(x_{s}+\bar{x}_{s}\right) \mathrm{d} s \\
& \geq \frac{1}{16}\|T x\| .
\end{aligned}
$$

For $-\tau \leq t \leq 0, x \in K$, one has $(T x)(t)=0$. That is $T(K) \subset K$.

Then we have the following lemma.

Lemma 4. The operator $T: K \rightarrow K$ is completely continuous.

Proof. We can obtain the continuity of $T$ from the continuity of $f$. In face, suppose $x^{(n)}, x \in K$ and $\left\|x^{(n)}-x\right\| \rightarrow 0$ as $n \rightarrow \infty$, then we get

$$
\left\|x_{s}^{(n)}-x_{s}\right\|_{C}=\sup _{-\tau \leq \theta \leq 0}\left|x^{(n)}(s+\theta)-x(s+\theta)\right| \rightarrow 0, s \in[0,1] .
$$

Thus, for $t \in[-\tau, 1]$ we have from (5) and Lemma 3 that

$$
\begin{aligned}
\mid\left(T x^{(n)}\right)(t) & -(T x)(t) \mid \\
\leq & \max _{0 \leq s \leq 1}\left|f\left(x_{s}^{(n)}+\bar{x}_{s}\right)-f\left(x_{s}+\bar{x}_{s}\right)\right| \int_{0}^{1} G(1, s) r(s) \mathrm{d} s .
\end{aligned}
$$

This implies that $\left\|T x^{(n)}-T x\right\| \rightarrow 0$ as $n \rightarrow \infty$.

Now let $\Omega \subset K$ be a bounded subset of $K$ and $M_{1}>0$ is the constant such that $\|x\| \leq M_{1}$ for $x \in \Omega$. Suppose that $\|\bar{x}\|=M_{2}$, then $\|x+\bar{x}\| \leq$ 
$M_{1}+M_{2}=: M$ for $x \in \Omega$. Define a set $S=\left\{\varphi \in C^{+} ;\|\varphi\|_{C} \leq M\right\}$. Let $L=\max _{0 \leq t \leq 1, \varphi \in S}|f(\varphi)+1|$, then we have

$$
\|T x\| \leq L \int_{0}^{1} G(1, s) r(s) \mathrm{d} s<+\infty,
$$

which implies the boundedness of $T(\Omega)$. Furthermore, we have for $0 \leq t \leq 1$,

$$
\begin{aligned}
(T x)^{\prime}(t) & =\frac{t^{\alpha-2}}{\Gamma(\alpha-1)} \int_{0}^{1}(1-s)^{\alpha-2} r(s) f\left(x_{s}+\bar{x}_{s}\right) \mathrm{d} s \\
& -\frac{1}{\Gamma(\alpha-1)} \int_{0}^{t}(t-s)^{\alpha-2} r(s) f\left(x_{s}+\bar{x}_{s}\right) \mathrm{d} s .
\end{aligned}
$$

So,

$$
\left|(T x)^{\prime}(t)\right| \leq \frac{2 L}{\Gamma(\alpha-1)} \int_{0}^{1}(1-s)^{\alpha-2} r(s) \mathrm{d} s=: L_{1}
$$

For $-\tau<t<0$, we have $(T x)^{\prime}(t)=0$. Thus with $x \in \Omega, \forall \epsilon>0$, let $\delta=\frac{\epsilon}{L_{1}}$, for $t_{1}, t_{2} \in[-\tau, 0],\left|t_{1}-t_{2}\right|<\delta$, we get $\left|(T x)\left(t_{1}\right)-(T x)\left(t_{2}\right)\right| \leq L_{1}\left|t_{1}-t_{2}\right|<\epsilon$. By means of the Arzela-Ascoli theorem, $T: K \rightarrow K$ is completely continuous.

Lemma 5. ([12]) Let $X$ be a Banach space, and let $K \subset X$ be a cone in $X$. Assume that $\Omega_{1}, \Omega_{2}$ are open subsets of $X$ with $0 \in \Omega_{1} \subset \bar{\Omega}_{1} \subset \Omega_{2}$, and let $T: K \rightarrow K$ be a completely continuous operator such that, either

(I) $\|T x\| \leq\|x\|, \forall x \in K \bigcap \partial \Omega_{1}$ and $\|T x\| \geq\|x\|, \forall x \in K \bigcap \partial \Omega_{2}$, or

(II) $\|T x\| \geq\|x\|, \forall x \in K \bigcap \partial \Omega_{1}$ and $\|T x\| \leq\|x\|, \forall x \in K \bigcap \partial \Omega_{2}$

holds. Then $T$ has a fixed point in $K \bigcap\left(\bar{\Omega}_{2} \backslash \Omega_{1}\right)$.

\section{Main Results}

For convenience, we introduce the following notations.

$$
\begin{gathered}
f_{0}=\limsup _{\|\varphi\|_{\mathrm{C}} \rightarrow 0} \frac{f(\varphi)}{\|\varphi\|_{C}}, \quad f_{\infty}=\limsup _{\|\varphi\|_{\mathrm{C}} \rightarrow+\infty} \frac{f(\varphi)}{\|\varphi\|_{C}}, \\
\bar{f}_{0}=\liminf _{\varphi \in C^{*},\|\varphi\|_{\mathrm{C}} \rightarrow 0} \frac{f(\varphi)}{\|\varphi\|_{C}}, \quad \bar{f}_{\infty}=\liminf _{\varphi \in C^{*},\|\varphi\|_{\mathrm{C}} \rightarrow+\infty} \frac{f(\varphi)}{\|\varphi\|_{C}},
\end{gathered}
$$




$$
\begin{gathered}
A=\frac{1}{3}\left[\int_{0}^{1} G(1, s) r(s) \mathrm{d} s\right]^{-1}, \quad B=\left[\frac{1}{16} \int_{E} G(1, s) r(s) \mathrm{d} s\right]^{-1}, \\
p_{0}=\max _{-\tau \leq t \leq 0}|\phi(t)|, \quad \Omega_{\alpha}=\{x \in C[-\tau, 1] ;\|x\|<\alpha\} .
\end{gathered}
$$

In the next, we let $C^{*}=\left\{\varphi \in C^{+} ; 0<\gamma_{0}\|\varphi\|_{C} \leq \varphi(\theta), \theta \in[-\tau, 0]\right\}$, where $0<\gamma_{0} \leq \frac{1}{16}$ is a constant.

Theorem 6. Assume that one of the following conditions is satisfied:

$\left(H_{1}\right) \quad f_{0}<A, \quad \bar{f}_{\infty}>B \gamma_{0}^{-1}$ (particularly, $f_{0}=0, \quad \bar{f}_{\infty}=+\infty$ ), $\phi(t) \equiv 0$;

$\left(H_{2}\right) \quad \bar{f}_{0}>B \gamma_{0}^{-1}, \quad f_{\infty}<A$ (particularly, $\left.\bar{f}_{0}=+\infty, \quad f_{\infty}=0\right)$. Then BVP (1) has at least one positive solution.

Proof. Suppose that $\left(H_{1}\right)$ is satisfied. By $\phi(t) \equiv 0$, we know $\bar{x}_{t}=0, t \in$ $[0,1]$. From $f_{0}<A$, there is a $\rho_{1}>0$ such that $f(\varphi) \leq A\|\varphi\|_{C}, \varphi \in C^{+}, 0 \leq$ $\|\varphi\|_{C} \leq \rho_{1}$. For any $x \in K,\|x\|=\rho_{1}$, we deduce that $\left\|x_{s}\right\|_{C} \leq\|x\|=\rho_{1}, s \in$ $[0,1]$ and thus

$$
\begin{aligned}
0 \leq(T x)(t) & \leq A\left\|x_{s}\right\|_{C} \int_{0}^{1} G(1, s) r(s) \mathrm{d} s \\
& \leq A\|x\| \int_{0}^{1} G(1, s) r(s) \mathrm{d} s<\|x\|, \quad(0 \leq t \leq 1)
\end{aligned}
$$

which implies $\|T x\|=\|T x\|_{[0,1]}<\|x\|, \forall x \in K \bigcap \partial \Omega_{\rho_{1}}$.

Since $\bar{f}_{\infty}>B \gamma_{0}^{-1}$, there exists a $\rho_{2}>\rho_{1}$ such that $f(\varphi) \geq B \gamma_{0}^{-1}\|\varphi\|_{C}$, $\varphi \in C^{*},\|\varphi\|_{C}>\gamma_{0} \rho_{2}$. For any $x \in K,\|x\|=\rho_{2}$, we have

$$
\gamma_{0}\left\|x_{s}\right\|_{C} \leq \gamma_{0}\|x\| \leq \frac{1}{16}\|x\| \leq \min _{\frac{1}{4} \leq t \leq \frac{3}{4}} x(t) \leq x(t), \quad t \in\left[\frac{1}{4}, \frac{3}{4}\right], s \in E .
$$

which implies that $x_{s} \in C^{*}$ for $s \in E$ and

$$
\left\|x_{s}\right\|_{C} \geq \gamma_{0}\|x\|=\gamma_{0} \rho_{2}, \quad s \in E
$$

Note that when $s \in E$ we have $\bar{x}_{s}=0$. Thus, we obtain

$$
\begin{aligned}
(T x)\left(\frac{1}{2}\right) & =\int_{0}^{1} G\left(\frac{1}{2}, s\right) r(s) f\left(x_{s}\right) \mathrm{d} s \geq \frac{1}{16} \int_{E} G(1, s) r(s) f\left(x_{s}\right) \mathrm{d} s \\
& \geq \frac{1}{16} B \gamma_{0}^{-1}\left\|x_{s}\right\|_{C} \int_{E} G(1, s) r(s) \mathrm{d} s \\
& \geq \frac{1}{16} B \gamma_{0}^{-1} \gamma_{0} \rho_{2} \int_{E} G(1, s) r(s) \mathrm{d} s=\rho_{2}=\|x\|,
\end{aligned}
$$

which leads to $\|T x\| \geq\|x\|, \forall x \in K \bigcap \partial \Omega_{\rho_{2}}$. According to the first part of Lemma 5 , it follows that $T$ has a fixed point $x \in K \cap\left(\bar{\Omega}_{\rho_{2}} \backslash \Omega_{\rho_{1}}\right)$ such that $0<\rho_{1} \leq\|x\| \leq \rho_{2}$. 
Now, suppose that $\left(H_{2}\right)$ is satisfied. Since $\bar{f}_{0}>B \gamma_{0}^{-1}$, there is a $\rho_{1}>0$ such that $f(\varphi) \geq B \gamma_{0}^{-1}\|\varphi\|_{C}, \varphi \in C^{*},\|\varphi\|_{C} \leq \rho_{1}$. For $x \in K,\|x\|=\rho_{1}$, we have $\left\|x_{s}\right\|_{C} \leq\|x\|=\rho_{1}, s \in[0,1]$. Furthermore, by a similar argument as (6), we have $x_{s} \in C^{*},\left\|x_{s}\right\|_{C} \geq \gamma_{0}\|x\|=\gamma_{0} \rho_{1}, s \in E$. Thus, we have an analogous result to $(7):(T x)\left(t_{1}\right) \geq \rho_{1}=\|x\|$, which implies that $\|T x\|=\|T x\|_{[0,1]} \geq$ $\|x\|, \forall x \in K \bigcap \partial \Omega_{\rho_{1}}$.

On the other hand, since $f_{\infty}<A$, there is a $N>\rho_{1}$ such that $f(\varphi) \leq$ $A\|\varphi\|_{C}, \varphi \in C^{+},\|\varphi\|_{C}>N$. Choose a positive constant $\rho_{2}$ such that

$$
\rho_{2}>\max \left\{p_{0}, 3 \max \left\{f(\varphi) ; 0 \leq\|\varphi\|_{C} \leq N+p_{0}\right\} \int_{0}^{1} G(1, s) r(s) \mathrm{d} s\right\} .
$$

For $x \in K,\|x\|=\rho_{2}$, we have from the facts: $\bar{x}(t) \geq 0, x(t) \geq 0, t \in[-\tau, 1]$, that for $s \in[0,1]$,

$$
\begin{aligned}
& \left\|x_{s}+\bar{x}_{s}\right\|_{C} \geq\left\|x_{s}\right\|_{C}>N, \quad\left\|x_{s}\right\|_{C}>N ; \\
& \left\|x_{s}+\bar{x}_{s}\right\|_{C} \leq\left\|x_{s}\right\|_{C}+\left\|\bar{x}_{s}\right\|_{C} \leq N+\|\bar{x}\|, \quad\left\|x_{s}\right\|_{C} \leq N .
\end{aligned}
$$

Thus, we have

$$
\begin{aligned}
(T x)(t) & \leq \int_{\left\|x_{\mathrm{s}}\right\|_{\mathrm{c}}>N} G(1, s) r(s) f\left(x_{s}+\bar{x}_{s}\right) \mathrm{d} s \\
& +\int_{0 \leq\left\|x_{\mathrm{s}}\right\|_{\mathrm{c}} \leq N} G(1, s) r(s) f\left(x_{s}+\bar{x}_{s}\right) \mathrm{d} s \\
& \leq A(\|x\|+\|\bar{x}\|) \int_{0}^{1} G(1, s) r(s) \mathrm{d} s \\
& +\max \left\{f(\varphi) ; 0 \leq\|\varphi\|_{C} \leq N+p_{0}\right\} \int_{0}^{1} G(1, s) r(s) \mathrm{d} s \\
& <\frac{1}{3}\|x\|+\frac{1}{3} p_{0}+\frac{1}{3} \rho_{2}<\rho_{2}=\|x\|, \quad 0 \leq t \leq 1,
\end{aligned}
$$

which implies that $\|T x\|<\|x\|, \forall x \in K \bigcap \partial \Omega_{\rho_{2}}$. By the second part of Lemma 5 , it follows that $T$ has a fixed point $x \in K \bigcap\left(\bar{\Omega}_{\rho_{2}} \backslash \Omega_{\rho_{1}}\right)$ such that $0<\rho_{1} \leq$ $\|x\| \leq \rho_{2}$.

Suppose that $x(t)$ is the fixed point of $T$ in $K \bigcap\left(\bar{\Omega}_{\rho_{2}} \backslash \Omega_{\rho_{1}}\right)$, then

$$
x(t)=\left\{\begin{array}{l}
\int_{0}^{1} G(t, s) r(s) f\left(x_{s}+\bar{x}_{s}\right) \mathrm{d} s, \quad 0 \leq t \leq 1, \\
0, \quad-\tau \leq t \leq 0 .
\end{array}\right.
$$

Let $u(t)=x(t)+\bar{x}(t)$. Since $0<\rho_{1} \leq\|x\|=\|x\|_{[0,1]} \leq \rho_{2}, x(t) \in K$ and $\bar{x}(t) \geq 0$, we conclude that $u(t)$ is a positive solution of $\operatorname{BVP}(1)$. This completes the proof. 
Theorem 7. If the following conditions are satisfied:

$\left(H_{3}\right) \quad \bar{f}_{0}>B \gamma_{0}^{-1}, \quad \bar{f}_{\infty}>B \gamma_{0}^{-1} \quad$ (particularly, $\left.\bar{f}_{0}=+\infty, \quad \bar{f}_{\infty}=+\infty\right)$; where

$\left(H_{4}\right) \quad \exists p>0$ such that for $\forall \varphi, \quad 0 \leq\|\varphi\|_{C} \leq p+p_{0}$, one has $|f(\varphi)|<\eta p$,

$$
\eta=\left(\int_{0}^{1} G(1, s) r(s) \mathrm{d} s\right)^{-1} .
$$

Then BVP (1)has at least two positive solution $u_{1}, u_{2} \in K$ such that $0<$ $\left\|u_{1}\right\|_{[0,1]}<p<\left\|u_{2}\right\|_{[0,1]}$.

Proof. Since $\bar{f}_{0}>B \gamma_{0}^{-1}$, there exists a $0<R_{1}<p$ such that $f(\varphi) \geq$ $B \gamma_{0}^{-1}\|\varphi\|_{C}, \varphi \in C^{*}, 0<\|\varphi\|_{C} \leq R_{1}$. For $x \in K,\|x\|=R_{1}$, similar to (6) one has $x_{s} \in C^{*}, R_{1} \geq\left\|x_{s}\right\|_{C} \geq \gamma_{0}\|x\|=\gamma_{0} R_{1}, s \in E$. Hence, we obtain an analogous inequality: $\|T x\| \geq\|x\|, \forall x \in K \bigcap \partial \Omega_{R_{1}}$.

Since $\bar{f}_{\infty}>B \gamma_{0}^{-1}$, there is a $R_{2}>p$ such that $f(\varphi) \geq B \gamma_{0}^{-1}\|\varphi\|_{C}, \varphi \in$ $C^{*},\|\varphi\|_{C} \geq \gamma_{0} R_{2}$. For $x \in K,\|x\|=R_{2}$, similar to (6) one has $x_{s} \in C^{*}$, $R_{2} \geq\left\|x_{s}\right\|_{C} \geq \gamma_{0}\|x\|=\gamma_{0} R_{2}, s \in E$. Hence, we obtain an analogous inequality: $\|T x\| \geq\|x\|, \forall x \in K \bigcap \partial \Omega_{R_{2}}$.

Now, by $\left(H_{4}\right)$, for $x \in K,\|x\|=p$, since $0 \leq\left\|x_{s}+\bar{x}_{s}\right\|_{C} \leq\left\|x_{s}\right\|_{C}+\left\|\bar{x}_{s}\right\|_{C} \leq$ $p+p_{0}$, we have

$$
\begin{aligned}
(T x)(t) & \leq \int_{0}^{1} G(1, s) r(s) f\left(x_{s}+\bar{x}_{s}\right) \mathrm{d} s \\
& \leq \eta p \int_{0}^{1} G(1, s) r(s) \mathrm{d} s=p=\|x\|, \quad 0 \leq t \leq 1,
\end{aligned}
$$

which implies that $\|T x\| \leq\|x\|, \forall x \in K \bigcap \partial \Omega_{p}$. According to Lemma 5, it follows that $T$ has two fixed points $x_{1}, x_{2}$ such that $x_{1} \in K \bigcap\left(\bar{\Omega}_{p} \backslash \Omega_{R_{1}}\right), x_{2} \in$ $K \cap\left(\bar{\Omega}_{R_{2}} \backslash \Omega_{p}\right)$, that is $0<\left\|x_{1}\right\|<p<\left\|x_{2}\right\|$. Since $x_{i}(t) \in K$, we have $x_{i}(t)>0, \forall t \in(0,1), i=1,2$. Let $u_{1}(t)=x_{1}(t)+\bar{x}(t), u_{2}(t)=x_{2}(t)+\bar{x}(t)$, then $u_{1}, u_{2}$ are positive solutions of $\operatorname{BVP}(1)$ satisfying $0<\left\|u_{1}\right\|_{[0,1]}<p<\left\|u_{2}\right\|_{[0,1]}$. This completes the proof.

Similarly, we have the following result.

Theorem 8. If the following conditions are satisfied:

$\left(H_{5}\right) \quad \bar{f}_{0}<A, \quad \bar{f}_{\infty}<A \quad$ (particularly, $\left.\bar{f}_{0}=0, \quad \bar{f}_{\infty}=0\right), \quad \phi(t) \equiv 0 ;$

$\left(H_{6}\right) \quad \exists p>0$ such that for $\forall \varphi, \gamma_{0} p \leq\|\varphi\|_{C} \leq p$, one has $|f(\varphi)| \geq B p$.

Then BVP (1)has at least two positive solution $u_{1} u_{2} \in K$ such that $0<$ $\left\|u_{1}\right\|_{[0,1]}<p<\left\|u_{2}\right\|_{[0,1]}$. 


\section{An Example}

Example 9. Consider the BVP

$$
\left\{\begin{array}{l}
D_{0^{+}}^{\frac{5}{2}} u(t)+\left(1+t^{2}\right) u^{\frac{1}{2}}\left(t-\frac{1}{3}\right)=0, \quad 0 \leq t \leq 1 \\
u(t)=-\sin \pi t, \quad-\frac{1}{3} \leq t \leq 0 \\
u(0)=u^{\prime}(0)=u^{\prime}(1)=0
\end{array}\right.
$$

Then $\tau=\frac{1}{3}, f(\varphi)=\varphi^{\frac{1}{2}}\left(-\frac{1}{3}\right), E=\left[\frac{7}{12}, \frac{3}{4}\right]$. As $\|\varphi\|_{C} \rightarrow+\infty$ we have

$$
\frac{f(\varphi)}{\|\varphi\|_{C}}=\frac{\varphi^{\frac{1}{2}}\left(-\frac{1}{3}\right)}{\|\varphi\|_{C}} \leq \frac{\|\varphi\|_{C}^{\frac{1}{2}}}{\|\varphi\|_{C}}=\|\varphi\|_{C}^{-\frac{1}{2}} \rightarrow 0
$$

that is to say that $f_{\infty}=0$ holds. On the other hand, suppose $\varphi \in C^{*}$, then $\varphi(\theta) \geq \gamma_{0}\|\varphi\|_{C}$, thus, as $\|\varphi\|_{C} \rightarrow 0$, we get

$$
\frac{f(\varphi)}{\|\varphi\|_{C}}=\frac{\varphi^{\frac{1}{2}}\left(-\frac{1}{3}\right)}{\|\varphi\|_{C}} \geq \frac{\gamma_{0}^{\frac{1}{2}}\|\varphi\|_{C}^{\frac{1}{2}}}{\|\varphi\|_{C}}=\gamma_{0}^{\frac{1}{2}}\|\varphi\|_{C}^{-\frac{1}{2}} \rightarrow+\infty,
$$

which means that $\bar{f}_{0}=+\infty$ holds. According to Theorem 6 , it follows that BVP (8) has at least a positive solution $u(t)$.

Example 10. Consider the BVP

$$
\left\{\begin{array}{l}
D_{0^{+}}^{\frac{5}{2}} u(t)+c\left[u^{\frac{1}{2}}\left(t-\frac{1}{3}\right)+u^{\frac{3}{2}}\left(t-\frac{1}{3}\right)\right]=0, \quad 0 \leq t \leq 1, \\
u(t)=\phi(t), \quad-\frac{1}{3} \leq t \leq 0 \\
u(0)=u^{\prime}(0)=u^{\prime}(1)=0 .
\end{array}\right.
$$

where $c>0$ is a constant, $\phi(t)$ is continuous on $\left[-\frac{1}{3}, 0\right], \phi(t) \geq 0, \phi(0)=0$ and $f(\varphi)=\varphi^{\frac{1}{2}}\left(-\frac{1}{3}\right)+\varphi^{\frac{3}{2}}\left(-\frac{1}{3}\right), \tau=\frac{1}{3}, E=\left[\frac{7}{12}, \frac{3}{4}\right]$. Suppose $\varphi \in C^{*}$, then $\varphi(\theta) \geq \gamma_{0}\|\varphi\|_{C}$ for $\theta \in\left[-\frac{1}{3}, 0\right]$ thus, as $\|\varphi\|_{C} \rightarrow 0$ or $\|\varphi\|_{C} \rightarrow+\infty$, we get

$$
\begin{aligned}
\frac{f(\varphi)}{\|\varphi\|_{C}} & =\frac{\varphi^{\frac{1}{2}}\left(-\frac{1}{3}\right)+\varphi^{\frac{3}{2}}\left(-\frac{1}{3}\right)}{\|\varphi\|_{C}} \\
& \geq \frac{\gamma_{0}^{\frac{1}{2}}\|\varphi\|_{C}^{\frac{1}{2}}+\gamma_{0}^{\frac{3}{2}}\|\varphi\|_{C}^{\frac{3}{2}}}{\|\varphi\|_{C}}=\gamma_{0}^{\frac{1}{2}}\|\varphi\|_{C}^{-\frac{1}{2}}+\gamma_{0}^{\frac{3}{2}}\|\varphi\|_{C}^{\frac{1}{2}} \rightarrow+\infty,
\end{aligned}
$$

Deducing $\eta=\left(\int_{0}^{1} G(1, s) r(s) \mathrm{d} s\right)^{-1}=\frac{45 \sqrt{\pi}}{16 c}$, then $\forall p>0$ and $0 \leq\|\varphi\|_{C} \leq p+p_{0}$, we have

$$
0 \leq f(\varphi) \leq\left(p+p_{0}\right)^{\frac{1}{2}}+\left(p+p_{0}\right)^{\frac{3}{2}}=\left(p+p_{0}\right)^{\frac{1}{2}}\left(1+\frac{1}{p}+\frac{p_{0}}{p}\right) p .
$$


Define $H(p)=\left(p+p_{0}\right)^{\frac{1}{2}}\left(1+\frac{1}{p}+\frac{p_{0}}{p}\right)$, then

$$
\lim _{p \rightarrow 0} H(p)=+\infty, \lim _{p \rightarrow+\infty} H(p)=+\infty .
$$

Suppose that $c$ and $p_{0}$ satisfy $\sqrt{p_{0}}\left(2+\frac{1}{p_{0}}\right)<\frac{45 \sqrt{\pi}}{16 \sqrt{2} c}$, then $H\left(p_{0}\right)=\sqrt{2} \sqrt{p_{0}}(2+$ $\left.\frac{1}{p_{0}}\right)<\frac{45 \sqrt{\pi}}{16 c}=\eta$ holds. By the continuity of $H(p)$ and $(10)$, we can find a $p>0$ such that $|f(\varphi)|<\eta p$ for $0 \leq\|\varphi\|_{C} \leq p+p_{0}$. By Theorem 7 we know that BVP (9) has at least two positive solutions.

\section{References}

[1] K.S. Miller, B. Ross, An introduction to the fractional calculus and fractional differential equation, Wiley-Interscience, USA (1993).

[2] K.B. Oldham, J. Spanier, The fractional Calculus, Academic Press, USA (1974).

[3] I. Podlubny, Fractional differential equations, Mathematics in Science and Engineering, Academic Press, USA (1999).

[4] V. Lakshmikantham, S. Leela, J. Vasundhara Devi, Theory of fractional Dynamic systems, Cambridge Academic Publishers, Cambridge (2009).

[5] V. Lakshmikantham, Theory of fractional functional differential equations, Nonlinear Analysis, 69 (2008), 3337-3343.

[6] Z.B. Bai, H.S. Lü, Positive solutions for boundary value problem of nonlinear fractional differential equation, J. Math. Anal. Appl., 311 (2005), 495-505.

[7] Z.B. Bai, T.T. Qiu, Existence of positive solution for singular fractional differential equation, Appl. Math. Comput., 215 (2009), 2761-2767.

[8] X. Liu, M. Jia, Multiple solutions for fractional differential equations with nonlinear boundary conditions, Comput. Math. Appl., 59 (2010), 28802886.

[9] S. Zhang, Positive solutions to singular boundary value problem for nonlinear fractional differential equation, Comput. Math. Appl., 59 (2010), 1300-1309. 
[10] L.M. Song, Existence of positive solutions to boundary value problem for a nonlinear fractional differential equations, Journal of South China Normal University: Natural Science Edition, 44 (2012), 25-28.

[11] L.M. Song, P.X. Weng, Existence of positive solutions for boundary value problem of fourth-order nonlinear functional differential equation, Applied Mathematics a Journal of Chinese Universities (Ser.A), 26 (2011), 67-77.

[12] M.A. Krasnosel'skii, Positive solutions of operator equations, P. Noordhoff, Netherlands (1964). 
